\title{
Examining the relationship between altered brain functional connectome and disinhibition across 33 impulsive and compulsive behaviours
}

Yann Chye, Chao Suo, Rafael Romero-Garcia, Richard A.I. Bethlehem, Roxanne Hook, Jeggan Tiego, Ian Goodyer, Peter B. Jones, Ray Dolan, Edward T. Bullmore, Jon E. Grant, Murat Yücel and Samuel R. Chamberlain

Impulsive and compulsive problem behaviours are associated with a variety of mental disorders. Latent phenotyping indicates the expression of impulsive and compulsive problem behaviours is predominantly governed by a transdiagnostic 'disinhibition' phenotype. In a cohort of 117 individuals, recruited as part of the Neuroscience in Psychiatry Network (NSPN), we examined how brain functional connectome and network properties relate to disinhibition. Reduced functional connectivity within a subnetwork of frontal (especially right inferior frontal gyrus), occipital and parietal regions was linked to disinhibition. Findings provide insights into neurobiological pathways underlying the emergence of impulsive and compulsive disorders.

\section{Keywords}

Impulsivity; compulsivity; brain network; connectome; transdiagnostic.

\section{Copyright and usage}

(c) The Author(s), 2021. Published by Cambridge University Press on behalf of the Royal College of Psychiatrists. This is an Open Access article, distributed under the terms of the creative Commons Attribution licence (http://creativecommons.org/ licenses/by/4.0/), which permits unrestricted re-use, distribution, and reproduction in any medium, provided the original work is properly cited.
It is increasingly acknowledged that psychiatric symptoms are underpinned by common latent constructs that manifest in an inappropriate or maladaptive manner. ${ }^{1}$ Specifically, 'impulsivity' and 'compulsivity' are two such critical constructs that typify a range of disorder presentation, e.g. substance dependence and obsessive-compulsive disorders. Impulsivity encompasses a predisposition toward poorly conceived actions, taking undue risks, and a lack of consideration when making decisions. ${ }^{2}$ Compulsivity is thought of as an inappropriate and maladaptive persistence of repetitive behaviour, undertaken according to rigid rules and/or as a means of avoiding perceived negative consequences. ${ }^{2}$ Although features of impulsive and/or compulsive behaviour exist in a dimensional fashion within the general population, reflecting a lack of control over thoughts and behaviour, more extreme pathological manifestations underpin a range of psychiatric disorders. ${ }^{2}$

Impulsive and compulsive problems are often examined separately as disparate constructs, but the cooccurrence and fluidity by which the two constructs may transition from one to the other suggest that some shared variance, and common brain mechanisms, contribute to both. ${ }^{2}$ Recent work demonstrated that $>70 \%$ of symptom expression across 33 impulsive and compulsive behaviours (as measured by the Impulsive-Compulsive Behaviours Checklist (ICBC)) could be statistically accounted for by a transdiagnostic 'disinhibition' phenotype. ${ }^{3}$ Conceptually, disinhibition reflects a lack of top-down executive control, reflected in both cognitive inflexibility-driven compulsivity and impulsivity. ${ }^{2}$ Yet, although a wealth of evidence documents brain network reorganisation across a range of impulsivity- and compulsivity-related mental disorders, compared with controls, little is known about network characteristics associated with disinhibition as viewed dimensionally along a continuum. Brain network dysregulation has been demonstrated to cut across diagnostic boundaries of psychiatric disorders, representing individual variability (e.g. impulsivity and compulsivity) that guide motivated behaviour. In this vein, deciphering the link between brain network topology and latent behavioural phenotypes (such as disinhibition) can be considered vital to clarifying the neurobiology of impulsive and compulsive disorders. ${ }^{2}$ The primary aim of this study was to use connectomics and graph theory to identify dysregulated brain networks associated with disinhibition, and therefore implicated as common substrate across impulsive and compulsive behaviours.

\section{Method}

Participants were recruited from a larger cohort of adolescents and young adults from the Neuroscience in Psychiatry Network (NSPN) study. ${ }^{4}$ A detailed description of the recruitment methods and sample have been previously published. ${ }^{4}$ In brief, the NSPN was set up as a demographically representative sample of the UK population, using a stratified recruitment design. Participants were entered on the basis of having no history of psychiatric treatment or neurological disorder, head injury or intellectual disability. Measures of impulsivity and compulsivity symptoms were collected with the ICBC, 3 years after enrolment. The ICBC assesses for the frequency of 33 common impulsive and compulsive problem behaviours (e.g. washing, smoking, gambling). ${ }^{3,5}$ In a previous paper, confirmatory factor analysis on 654 participants' ICBC response identified a single latent factor (termed 'disinhibition') accounting for around $70 \%$ of the variance in participants' ICBC scores (see Supplementary Table 1 available at https://doi.org/10.1192/bjp.2021.49, for ICBC checklist items and loadings). ${ }^{3}$

\section{Magnetic resonance imaging acquisition and preprocessing}

From the original NSPN cohort, a random subset of participants completed neuroimaging, of whom data were available from 117 who had also undertaken the subsequent round collecting the ICBC data. The study was approved by the Cambridge East Research Ethics Committee (approval number 207190), and individuals provided informed consent. 
(a)

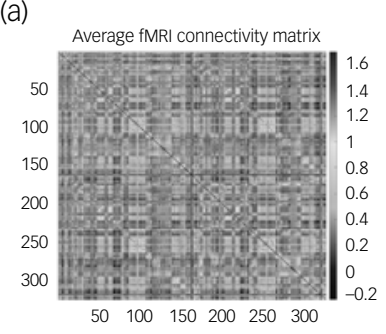

(C)

Left

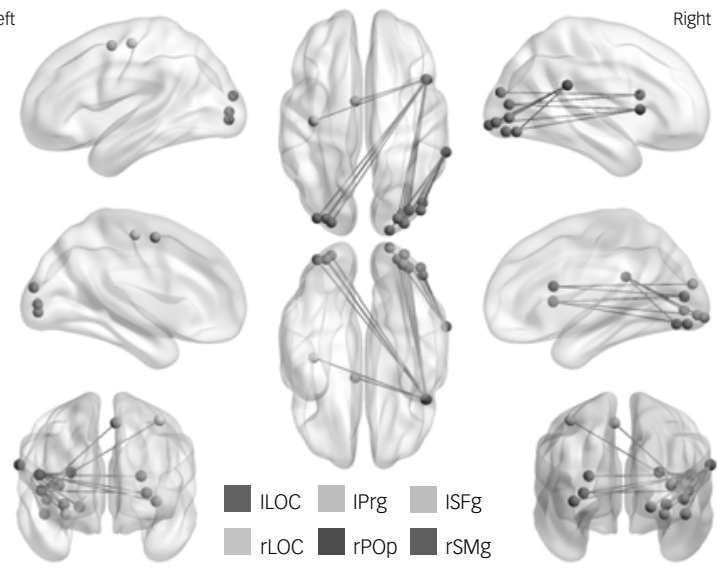

Fig. 1 (a) Average functional magnetic resonance imaging (fMRI) connectivity matrix. (b) Subnetwork showing reduced functional connectivity associated with latent disinhibition phenotype, assessed with the network-based statistics (NBS) software package and visualised with BrainNet Viewer. (c) The subnetwork consists of 15 edges across 15 regions, connecting the right pars opercularis (in deep blue) to bilateral lateral occipital regions (in red and light blue), the right lateral occipital region to the right supramarginal region (in pink), and the right pars opercularis to the left precentral (in yellow) and left superior frontal gyri (in green), respectively. ILOC, left lateral occipital; IPrg, left precentral gyrus; ISFg, left superior frontal gyrus; rLOC, right lateral occipital; rPOp, right pars opercularis; rSMg, right supramarginal.

Brain scans were conducted at three sites: two at the University of Cambridge (the Wolfson Brain Imaging Centre and the Medical Research Council Cognition \& Brain Sciences Unit) and one at University College London (the Wellcome Trust Functional Imaging Laboratory). All sites had identical 3 T, 32-channel magnetic resonance imaging (MRI) systems (Magnetom TIM Trio) and a unified acquisition sequence. Resting-state functional scans were obtained with a multi-echo echoplanar imaging sequence. Scanner detail and preprocessing steps can be found in the study published by Váša et al. ${ }^{6}$

\section{Parcellation and network-based statistics analysis}

Following preprocessing, functional MRI images were parcellated by subdividing the Desikan-Killiany anatomical atlas into 308 cortical parcels of approximately equal surface area (around $500 \mathrm{~mm}^{2}$ ) and 16 subcortical regions. Blood oxygenation level dependent time series were estimated from the average over all voxels within each of the 324 parcels (nodes). Pearson correlation was calculated between the time series of each pair of nodes, to determine their functional connectivity strength, resulting in a symmetric $324 \times 324$ connectivity matrix for each participant (Fig. 1). The resultant matrices were Fisher's r-to-z transformed to improve normality of the correlation estimates.
The network-based statistics connectome software package (version 1.2; https://www.nitrc.org/projects/nbs/) was used to assess for association between interregional connectivity matrix and disinhibition scores, controlling for age, gender and IQ. IQ was recorded with the Wechsler Abbreviated Scale of Intelligence - Second Edition (WASI-II) ${ }^{7}$. This first included mass univariate testing at each edge, with a primary component-forming threshold of $P<0.0001$ uncorrected. Each identified component (i.e. topologically connected subnetwork) was then assessed at 10000 permutations, using a family-wise error rate-corrected level of $P<0.05$.

\section{Graph theory analysis}

Graph theoretic analysis (i.e. modelling the brain network as a graph of interconnected regions/nodes) was further used to examine specific brain connection properties. The Brain Connectivity Toolbox ${ }^{8}$ was used, and properties examined include local network properties (nodal degree, normalised betweenness centrality, local efficiency and clustering coefficient) and global network properties (global efficiency and transitivity). These properties were examined across sparsity thresholds of 0.05 to 0.2 (at increments of 0.01 ). The areas under the curve across the threshold range for the listed properties were computed, and partial correlation was used to determine if any of these network properties were significantly associated with disinhibition, again controlling for age, gender and IQ. Local and global properties were assessed at $P<0.000154$ and $P<0.025$, respectively (i.e. Bonferroni-corrected for number of nodes and number of global properties, respectively).

\section{Results}

The 117 participants (71 women) had a mean age of 22.6 (s.d. 2.7, range 18-28) years, and mean IQ of 112.5 (s.d. 10.7). Disinhibition factor score estimates ranged from -1.38 to 2.28 (mean 0.05 , s.d. 0.81). Network-based statistics analysis revealed a subnetwork of 15 edges across 15 regions, which was significantly negatively associated with disinhibition scores (family-wise error rate-corrected $P=0.0203$ ). The connections primarily linked the right inferior frontal brain region (right pars opercularis) to bilateral lateral occipital regions ( 9 out of 15 connections/edges), and the right lateral occipital region to the right parietal (supramarginal) region (4 edges). Two other edges connect the right pars opercularis to the left precentral and left superior frontal gyri. See Fig. 1 for brain networks, visualised with BrainNet Viewer (version 1.7 for MacOS; https://www.nitrc.org/projects/bnv/). ${ }^{9}$ Examination of local (all $P>0.00039, r<0.32$ ) and global (all $P>0.016, r<0.23$ ) network metrics revealed no other significant association between network measures and disinhibition scores, at the Bonferroni correction threshold.

\section{Discussion}

This study demonstrates connectome-level variation in brain functional networks associated with disinhibition, providing the first evidence of common functional substrates contributing to both impulsive and compulsive behaviour problems. Reduced network connections across a subnetwork of frontal (specifically right inferior frontal), parietal and occipital regions were observed in association with increased disinhibition. Over half of the affected edges connect the bilateral occipital regions to the right inferior frontal region - specifically, the pars opercularis. These changes were regionally specific and did not reflect changes in global network properties. These data indicate that variation in connectivity is related to disinhibition, which was driven primarily by connection 
to the inferior frontal region (as measured by total number of connecting edges). This converges with other studies emphasising the central role of the inferior frontal brain region in response inhibition and maladaptive behavioural problems. ${ }^{2}$ The inferior frontal and occipital cortices are co-activated, and show marked functional coupling when inhibitory control is required. ${ }^{10}$ Additionally, reduced inhibition-related functional connectivity between frontal and posterior brain regions constitutes a candidate vulnerability marker for obsessive-compulsive disorder. ${ }^{11}$ The current data suggest that aberrant long-distance resting-state connections along the anterior-posterior axis may play a role in predisposing toward disinhibited behaviour.

\section{Limitations and conclusion}

The original NSPN cohort was recruited to be epidemiologically representative of the general UK population. This reduces selection biases inherent in clinical samples, but may limit the applicability of findings to those with more severe psychopathology (i.e. higher disinhibition). Another limitation is that the study shows association not causality. Future work should examine what precise underlying mechanisms (psychological and biological) contribute to the observed link between disinhibition and brain dysconnectivity. However, our findings provide insights into neurobiological processes that confer vulnerability to many types of problematic impulsive and compulsive behaviours, and therefore may be relevant to the search for transdiagnostic heuristics. Extending these techniques into patient populations, and larger imaging cohorts, will ideally refine our current understanding of the aetiology and course of psychiatric disorders, and the role of common latent phenotypes in the emergence of psychiatric conditions. This will require the inclusion of appropriate measurement tools for impulsivity and compulsivity in large-scale population studies, which have typically overlooked such dimensional measures in favour of binary measures or interrogation of symptoms of discrete disorders (e.g. obsessive-compulsive disorder).

Yann Chye (10), BrainPark, The Turner Institute for Brain and Mental Health, School of Psychological Sciences and Monash Biomedical Imaging Facility, Monash University, Australia; Chao Suo, BrainPark, The Turner Institute for Brain and Mental Health, Schoo of Psychological Sciences and Monash Biomedical Imaging Facility, Monash University, Australia; Rafael Romero-Garcia, Department of Psychiatry, University of Cambridge, UK; Richard A.I. Bethlehem, Department of Psychiatry, University of Cambridge, UK; Roxanne Hook, Department of Psychiatry, University of Cambridge, UK; Jeggan Tiego Neural Systems and Behaviour Laboratory, The Turner Institute for Brain and Mental Health, School of Psychological Sciences and Monash Biomedical Imaging Facility, Monash University, Australia; Ian Goodyer (D). Department of Psychiatry, University of Cambridge, UK; Peter B. Jones $(\mathbb{D}$, Department of Psychiatry, University of Cambridge UK; Ray Dolan, Max Planck University College London Centre for Computational Psychiatry and Ageing Research, UK; Wellcome Centre for Human Neuroimaging, University College London, UK; Edward T. Bullmore, Department of Psychiatry, University of Cambridge, UK; Jon E. Grant $(\mathbb{D}$, Department of Psychiatry, University of Chicago, USA; Murat Yücel, BrainPark, The Turner Institute for Brain and Mental Health, School of Psychological Sciences and Monash Biomedical Imaging Facility, Monash University, Australia; Samuel R. Chamberlain (D), Department of Psychiatry, University of Cambridge, UK; Department of Psychiatry, Faculty of Medicine, University of Southampton, UK; Southern Health NHS Foundation Trust, UK; and Cambridgeshire \& Peterborough NHS Foundation Trust, UK

Correspondence: Sam Chamberlain. Email: srchamb@gmail.com

First received 14 Sep 2020, final revision 20 Jan 2021, accepted 17 Mar 2021

\section{Supplementary material}

To view supplementary material for this article, please visit https://doi.org/10.1192/bjp.2021.49

\section{Data availability}

The data that support the findings of this study are available from the corresponding author, Y.C., upon reasonable request.

\section{Author contributions}

R.R-G, R.A.IB, R.H. I.G., P.B.J., R.D., E.T.B., J.E.G., M.Y. and S.R.C. were responsible for study conceptualisation and data collection. Y.C., M.Y. and S.R.C. formulated the specific research question and contributed to the interpretation of data. Y.C., C.S., R.R-G. and J.T. conducted the analysis. Y.C. drafted the paper, with support and input from all authors.

\section{Funding}

This research was funded by a clinical fellowship from the Wellcome Trust to S.R.C. (reference $110049 / 2 / 15 / Z$ \&/A). The study was supported by the Neuroscience in Psychiatry Network, a strategic award from the Wellcome Trust to the University of Cambridge and University College London (reference 095844/Z/11/Z); and by the National Institute for Health Research (NIHR) Cambridge Biomedical Research Centre (Mental Health).

\section{Declaration of interest}

Y.C. was supported by National Health and Medical Research Council (NHMRC) project grant 1139979. R.R.-G. was funded by a Guarantors of Brain fellowship. E.T.B. is an NIHR Senior Investigator. R.A.I.B. was funded by a British Academy postdoctoral fellowship. S.R.C. consults for leso Digital Health, and receives stipends from Elsevier for editorial work at Comprehensive Psychiatry and Neuroscience \& Biobehavioral Reviews. J.E.G. has received research grants from the National Institute on Drug Abuse (NIDA), National Center for Responsible Gaming, American Foundation for Suicide Prevention, Forest Pharmaceuticals and Roche Pharmaceuticals; receives yearly compensation from Springer Publishing for acting as Editor-in-Chief of the Journal of Gambling Studies; and has received royalties from Oxford University Press, American Psychiatric Publishing Norton Press, Johns Hopkins University Press and MCGraw Hill. J.T. was supported by NHMRC project grants 1002458 and 1046054. I. G. consults for Lundbeck; is supported by a Wellcome Trust Strategic Award; and is Chairperson of and scientific advisor to the Peter Cundill Centre for Youth Depression Research, Centre for Addictions and Mental Health, University of Toronto. E.T.B. is a member of the Sosei Heptares scientific advisory board and is a National Institute of Health Research Senior Investigator. M.Y. has received funding from Monash University and Australian Government funding bodies such as the NHMRC (including fellowship APP1117188), the Australian Research Council, Australian Defense Science and Technology, and the Department of Industry, Innovation and Science. He has also received philanthropic donations from the David Winston Turner Endowment Fund and the Wilson Foundation; and payment from law firms in relation to court, expert witness and/or expert review reports. The funding sources had no role in the design, management, data analysis, presentation, interpretation or write-up of the data. The other authors report no disclosures or potential conflicts of interest.

\section{References}

1 Cuthbert BN, Insel TR. Toward the future of psychiatric diagnosis: the seven pillars of RDoC. BMC Med 2013; 11: 126.

2 Fineberg NA, Chamberlain SR, Goudriaan AE, Stein DJ, Vanderschuren LMJ, Gillan CM, et al. New developments in human neurocognition: clinical, genetic, and brain imaging correlates of impulsivity and compulsivity. CNS Spectr 2014; 19(1): 69-89.

3 Chamberlain SR, Tiego J, Fontenelle LF, Hook R, Parkes L, Segrave R, et al. Fractionation of impulsive and compulsive trans-diagnostic phenotypes and their longitudinal associations. Aust N Z J Psychiatry 2019; 53(9): 896-907.

4 Kiddle B, Inkster B, Prabhu G, Moutoussis M, Whitaker KJ, Bullmore ET, et al. Cohort profile: the NSPN 2400 Cohort: a developmental sample supporting the Wellcome Trust NeuroScience in Psychiatry Network. Int J Epidemiol 2018; 47(1): 18-9g.

5 Guo K, Youssef GJ, Dawson A, Parkes L, Oostermeijer S, López-Solà C, et al A psychometric validation study of the Impulsive-Compulsive Behaviours Checklist: a transdiagnostic tool for addictive and compulsive behaviours. Addict Behav 2017; 67: 26-33.

6 Váša F, Romero-Garcia R, Kitzbichler MG, Seidlitz J, Whitaker KJ, Vaghi MM, et al. Conservative and disruptive modes of adolescent change in human brain functional connectivity. Proc Natl Acad Sci U S A 2020; 117(6): 3248-53.

7 Wechsler D. Wechsler Abbreviated Scale of Intelligence (2nd ed WASI-II). San Antonio, TX: NCS Pearson, 2011.

8 Rubinov M, Sporns O. Complex network measures of brain connectivity: uses and interpretations. Neuroimage 2010; 52(3): 1059-69.

9 Xia M, Wang J, He Y. BrainNet viewer: a network visualization tool for human brain connectomics. PLOS One 2013; 8(7): e68910.

10 Tsvetanov KA, Ye Z, Hughes L, Samu D, Treder MS, Wolpe N, et al. Activity and connectivity differences underlying inhibitory control across the adult life span. J Neurosci 2018; 38(36): 7887-900.

11 Hampshire A, Zadel A, Sandrone S, Soreq E, Fineberg N, Bullmore ET, et al. Inhibition-related cortical hypoconnectivity as a candidate vulnerability marker for obsessive-compulsive disorder. Biol Psychiatry Cogn Neurosci Neuroimaging 2020; 5(2): 222-30. 